\title{
Topos: Spiking Neural Networks for Temporal Pattern Recognition in Complex Real Sounds
}

\author{
Pablo González-Nalda *,1 Blanca Cases*
}

\begin{abstract}
This article depicts the approach used to build the Topos application, a simulation of two-wheel robots able to discern real complex sounds. Topos is framed in the nouvelle concept of subsymbolic artificial intelligence, applied to the field of evolutionary robotics. This paper focuses on the simulation of biologically inspired artificial cochleas and spiking neural networks, in order to model the embodied control system of the robots. The method chosen to find the most appropriate parameters that determine robots' behaviour is evolutionary computation techniques, with the aim of avoiding any human intervention in this task. As an example of a real application of this technique, experiments were performed to study the ability of the robots to distinguish sounds composed of parts of real canary songs and to navigate to the recognised signal. Results obtained confirm the validity of the approach.
\end{abstract}

Key words: Evolutionary Robotics, Spiking Neural Networks, Sound Perception, Sensorimotor Transformations, Auditory Navigation

\section{Introduction}

This article depicts the interdisciplinary field that results from applying basic Neuroscience approaches to a technical discipline known as Evolutionary

*

Computer Languages and Systems, University of the Basque Country (UPV/EHU)

Email address: pablo@si.ehu.es (Pablo González-Nalda).

URL: http://lsi.vc.ehu.es/pablogn/ (Pablo González-Nalda).

1 The work that produced this article was supported by the UPV-EHU project

9/UPV 00003.230-15840/2004.

We also thank Endika Bengoetxea for his help. 
Robotics (described in Subsection 2.3). This work shows that it is possible to employ evolutionary computation techniques in order to optimise the neural network that leads to a desired behaviour of simulated robots. This approach provides more biologically inspired solutions for solving some engineering problems. Sometimes these techniques are the only way to solve some problems with no analytical solution. This is an important issue in the evolutionary robotics field since it is considered as the first step in the development of real autonomous robots (i.e. not human controlled) that are able to choose and exhibit a concrete behaviour. As an additional contribution of this approach, the results obtained have the potential to validate further theoretical proposals on Neuroscience and on Biology.

Genetic Algorithms (see Subsection 2.1) are applied to evolve (in a darwinist sense) a population of individuals. Each individual is described by a set of parameters. The evolution tunes both the sensors and the neural network that control the behaviour of virtual robots by changing the parameters. These robots capture sounds through the sensors, apply the Fourier Transform to the received signals, and their Spiking Neural Network process the stimulus to activate the motors required to exhibit a chosen behaviour. All the parameters that define the behaviour of each robot (i.e. that tune the sensors and the neural network) are encoded into the genome of an individual, and a Genetic Algorithm is applied to obtain the best parameters that respond to a desired behaviour of the robots.

The problem consisting on the navigation through recognition of sound landmarks serves to test the approach, implemented by an application called Topos. This problem allows to use the paradigm defined as Nouvelle Artificial Intelligence, where there are no boundaries between Sense, Plan and Act phases.

The sense of hearing was chosen instead of others due to the existence of less dimensions for sounds rather than for spatial signals like light or for the sense of touch. This decision facilitates the simulation process without loosing richness in the signals. Sound landmarks are an example of cues that can be obtained when observing a non-structured environment, and the navigation with landmarks is crucial for robots to be able to accomplish their tasks. Such a goal requires recognition of time patterns in signals that come from some sensors, which at the same time send activation signals to motors to exhibit a behaviour. The part of the robots in charge of this recognition task and consequent motor activation is called the control system of the robot.

The Topos application ${ }^{2}$ is a computational model that applies evolutionary computation techniques to search for the best control system parameters under

$\overline{2}$ Topos means place in Greek, and here it reflects the concept of navigation 
a concrete environment. The evaluation of each control system is made by simulating a rough abstraction of a two-wheel robot and its environment. The difficulties of a realistic simulation (inertia, motor noises...) are not taken into account for the sake of simplicity, nor the hunger of computational resources of the simulation, in order to facilitate and improve the analysis and the increase the number of experiments.

The behaviour searched for in our studies is the following: given two different real canary sounds, the virtual robot has to differentiate them and to approach the place where one of the sounds is being emitted. This scheme allows to know whether the robot discriminates the correct signal and shows its ability to move towards the source. Other sounds than canary songs could have been chosen from a wide range of real samples such as music or animal sounds, or they could also be generated, like white or pink noise. The song of birds was chosen since it is decomposable in three different parts separated by silence, and allows to compose two sequences that differ only in a twist of the last two parts (ABC-ACB like order). Computational overhead makes it necessary to limit the sound length and the number of parts of the birdsong used to compose the sound. At first, the problem described might be considered trivial or too simple as to be interesting, although so far the literature in the evolutionary robotics field does not provide any work able to accomplish a similar task with complex and dynamic signals.

In most cases the models in Computational Neuroscience perform abstractions and test models of single neurons with random inputs, and often lack a whole-agent dimension [31]. This work in evolutionary robotics tries to fill the gap between the abstract models in Neuroscience and the use of neural networks such as a robot controller. This application is based on a embodied and situated bio-inspired neuronal model that carries out a complex signal recognition task. The inputs and outputs are linked through the sensorimotor loop, and they have to deal with analog time-signals.

The outline of this work is as follows: the following section presents some topics related to Artificial Intelligence (AI) and Robotics, as the basics of Genetic Algorithms and the way of modelling phonotaxis (Subsection 2.1). Subsection 2.2 draws on the two different perspectives for AI, and Subsection 2.3 describes the context of evolutionary robotics within AI.

Section 3 describes the design of the agents and the representation of the environment of Topos system. Subsections 3.2 and 3.3 are devoted to the description of the auditory system and the spiking neural networks for temporal pattern recognition in complex sounds, respectively.

The next section presents the experimental results. Experimental data in Subsection 4.1 proves the correction of Topos when stimulating with simple 
signals. Subsections 4.2 and 4.3 describes experiments that corroborate the capacity of the system in the distinction between real canary songs and the robustness to background sounds and noises, respectively. Finally, the conclusions show some considerations and suggest some lines of development and application of this work.

\section{Artificial Intelligence and Robotics}

This section presents some AI techniques and concepts that determine the approach used to face this work in Robotics.

\subsection{Genetic Algorithms and Braitenberg Vehicles}

This work evolves a population of virtual robots with the help of genetic algorithms, a well-known paradigm in AI to solve optimization problems as heuristic search in a space of states [1-3]. As in any evolutionary computation technique, a pool or set (population) of possible solutions (individuals, and in this case, robots) is maintained for a concrete problem. The traditional way to represent solutions is in binary, as strings of $0 \mathrm{~s}$ and $1 \mathrm{~s}$, but in this work and others, the encoding uses an array of real numbers that describes the characteristics that can vary.

All the individuals are evaluated with a fitness function that quantifies the correctness of each solution for solving the problem. When using the elitist approach, the best individuals from each generation are kept and the rest are discarded. Some individuals survive to the next generation without changes (the elite), and the rest of individuals (used as parents) undergo a crossover and mutation operators. These genetic operators create other possible solutions (mixing the bit strings that represent their parents) that are hoped to be more promising in order to improve inherited attributes. The iteration of this process of evaluation, selection and mix, forces the population to improve the fitness values.

In this paper, the concept of phonotaxis and the navigation with sound landmarks is implemented with the help of Braitenberg Vehicles [4]. Braitenberg vehicles are thought experiments based on tropism and taxis: the movements of plants and animals toward or away from a stimulus. The Braitenberg vehicles that exhibit positive taxis are symmetric devices composed of two frontal sensorial inputs together with a free wheel and two back wheels propelled by motors. The vehicle is governed by a circuit that makes a crossed connection from the left sensor to the right motor and from the right sensor to the left 
motor. If the left sensor is fired, the right motor speeds up and the vehicle runs turning to the left; when the left sensor receives the signal, the vehicle advances turning to the right, until the vehicle reaches the source of the stimulus.

\subsection{The two approaches to Artificial Intelligence}

In the last two decades, AI has entered upon a veritable revolution due to the influence of new fields such as artificial life, which provided the conceptual framework for this work.

Artificial life (AL) is the study of artificial systems in which we can observe an analogous functioning to that of real biological systems $[5,6]$. This is complementary to AI because one of the aims of AL is intelligence, but in a bottom-up and incremental way. Another goal of AL is the simulation of adaptive and life-like behaviours [7].

Important changes in the classical foundations of robotics in AI come from some works in which Brooks affirms that intelligence requires situatedness and embodiment [8-10]. A system is situated when it does not deal with abstract descriptions of the world. The agent must operate within the real world and cope with time problems. An embodied system depends on the influence of its own physical body interacting with the environment. Brooks also describes two approaches to artificial intelligence:

Classical AI: founded on the symbol system hypothesis, it is based on the assumption that the outputs of sensors are symbols, so the reasoning system is built apart isolated from sensors and motors [3]. This scheme is known as the Sense-Plan-Act model [9]. The system works in a domain independent way, and only when the observer assigns the meaning to the symbols the whole system is grounded and makes sense ${ }^{3}$.

Nouvelle AI: based on the physical grounding hypothesis. The system has to build its representations grounded on the physical world. The world is its own best model. Following this, the system has to be built in a bottom-up manner. The meaning of every part of the system lies on the environment through interfaces, sensors and motors. Symbols and representation are not needed, or at least the designer does not have to define symbols, that is, there are no human generated symbols. The symbols of the system emerge from the system dynamics, and are not defined in design.

A situated agent can be described as a dynamical system coupled with its

$\overline{3}$ As Brooks says "[central] representations are not necessary and appear only in the eye or mind of the observer" [9] and "Intelligence is in the eye of the observer" [10] 
environment [11]. The agent can also be defined as a dynamical control system coupled with the body. The body is considered as a dynamical system because of its physical dynamics. Hence, a natural way to implement situatedness is by means of dynamical artificial neural networks. If the system is physically grounded, the system structure links the world and the actions of perception, planning and action in a continuum. It is impossible to set boundaries among them, in contrast to the Sense-Plan-Act scheme, where explicit modules and interfaces are defined.

\subsection{Artificial Intelligence and Evolutionary Robotics}

The work presented in this paper is at the cross-point of AI and artificial life, in the nouvelle conception of artificial intelligence, applying techniques of evolutionary robotics. Efforts were concentrated on developing a biologically inspired simulation of robots. The main parameters that characterise a two-wheel robot are taken into account in order to obtain a real embodied and situated robot in a further development of this work. In this bio-inspired framework, spiking neural networks are applied to model the control system of the robots.

It is difficult to design control systems for autonomous robots by hand, especially if the work applies an emergent scheme of subsymbolic systems [9]. Some works propose the use of Genetic Algorithms to achieve the desired behaviour (in our work, navigation through landmark recognition) with minimal human design [12-14]. This technique is called evolutionary robotics, a young discipline based on the work developed by Brooks [8], Koza [15], Beer and Gallagher [16], Floreano and Mondada [17], and Harvey, Husbands and Cliff [18]. The term evolutionary robotics was introduced by Peter Cariani in 1987 in a unpublished work, as explained by Harvey et al. [19].

To sum up, evolutionary robotics is the development of the control system of a robot (in simulation or not) testing possible robot configurations and choosing the fittest ones in order to form the next generation of solutions, until one or some robots perform the task correctly. As the task can resemble the behaviour of a living being, we also use the word behaviour to name the task.

\section{Topos}

The Topos application was introduced for the first time in [20,21]. Topos evolves, with the help of Genetic Algorithms, a population of virtual robots 
in a simulation to make them capable of recognising sounds.

\subsection{The problem}

Each robot is evaluated and assigned a fitness value according to the ability of recognising and choosing one of a total of two different sounds. The problem with two sounds is the simplest one for the system to be defined, and also for allowing to analyse whether the robot recognises sounds correctly. A robot passes to the next generation if it performs well in a number of trials in a skinner box-like scheme [22]. The robot has to reach the source of a sound and avoid the other source, choosing the correct one by recognition of sounds. Which sound is the goal is determined for each experiment (the whole run of the genetic algorithm) by the fitness function. The roubots use sounds as navigation landmarks.

This problem links the sound recognition to the motor system of the robots. The robot has to recognise a temporal pattern in order to choose the right source (perception), and it has to navigate (action) to that source by spatially locating and isolating it perceptually from the other source. This is important because it is consistent with the approach based on Nouvelle AI and the claim that the Sense-Plan-Act model is not necessary.

The fitness function defines a landscape in the state space. If it is not too rough the solution can evolve so to climb to local or global maxima, since the fitness value is closely related to the mean ability to solve the problem, that is, the robots get closer and closer through generations.

"Learning" in the skinner-box occurs with phylogenetic adaptation. The reinforcement of the appropriate innate behaviour is the survival of the individual or the heredity of the behaviour to its offspring, and the behaviour that does not give adaptation to the problem is not inherited. The robot does not change in any value during the evaluation. There is no hebbian learning nor other type of ontogenetical adaptation through modifications of the structure of the robot, that remains as the codified in the genome (numerical description of the virtual robot). Recombination and mutation are the tools that create new individuals that can perform better or not.

In each trial the correct sound is randomly placed in one of two points that are equidistant to the robot, so the correct sound can be on the right or on the left. The other sound is placed in the remaining place. For each trial a score is calculated. At the end of the trials the fitness value for this robot is the sum of the scores. The score of a trial is initially of 10000 units. The minimal distance $d$ to the correct source and $\bar{d}$ to the wrong source is calculated for each test as the robot moves and gets closer or not. See the figure 1 for some examples of 


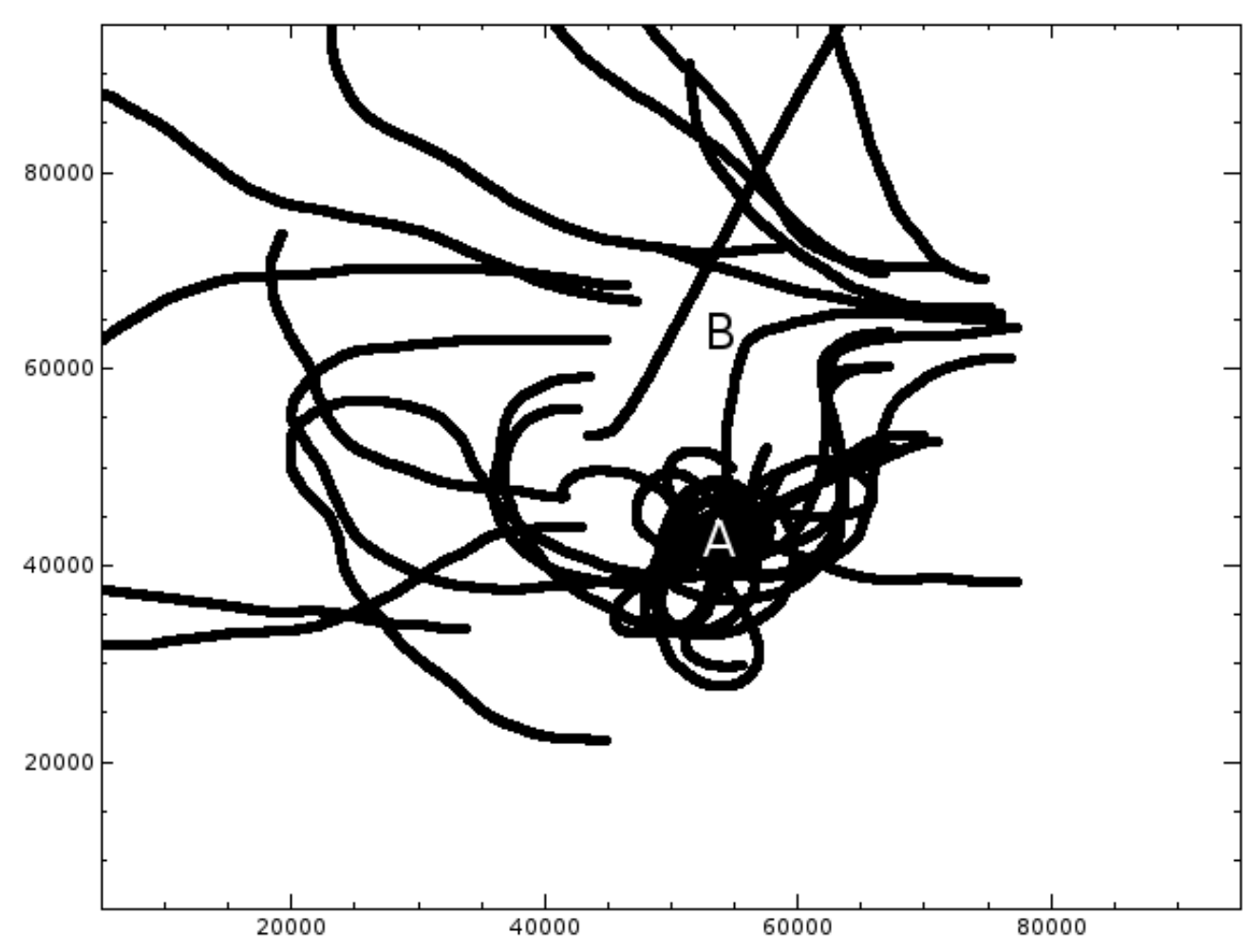

Fig. 1. Spatial trajectories from some start points for a developed robot that instinctively gets closer to the sound coming from the source A and avoids sound from B

trajectories performed by a evolved robot that distinguishes the sound from the figure 9 and can reach the correct source A and avoid the wrong one, in spite of the starting point.

The fitness of the individual is incremented (or decremented) by $10\left(\bar{d}^{2}-d^{2}\right)$. The greater the score, the better the robot has performed. If the robot reaches the target zone of the correct source (hit), a bonus of 8000 units is added. Symmetrically, 8000 points are decremented when the robot reaches the wrong source (miss). This fitness value is an indirect measure of the behaviour of a robot and its a neural network and sensors. If the individual performs well, its spiking neural network is appropriate to the task, and no other value is necessary for the genetic algorithm to function.

An elite (the individuals with highest scores in the test, see Section 2.1) is selected for survival to the next generation (25\%) and the other places in the fixed size population (75\%) are filled with crossover of two parents. Parents are chosen from the past population (elite included) with a uniform probability, excluding the worst fitted individuals from the lower fourth of the ranking (25\%). The optimization of fitness values can be seen in the figure 2 .

The simulation of these two-wheel robots is symmetric by design [23], in order 


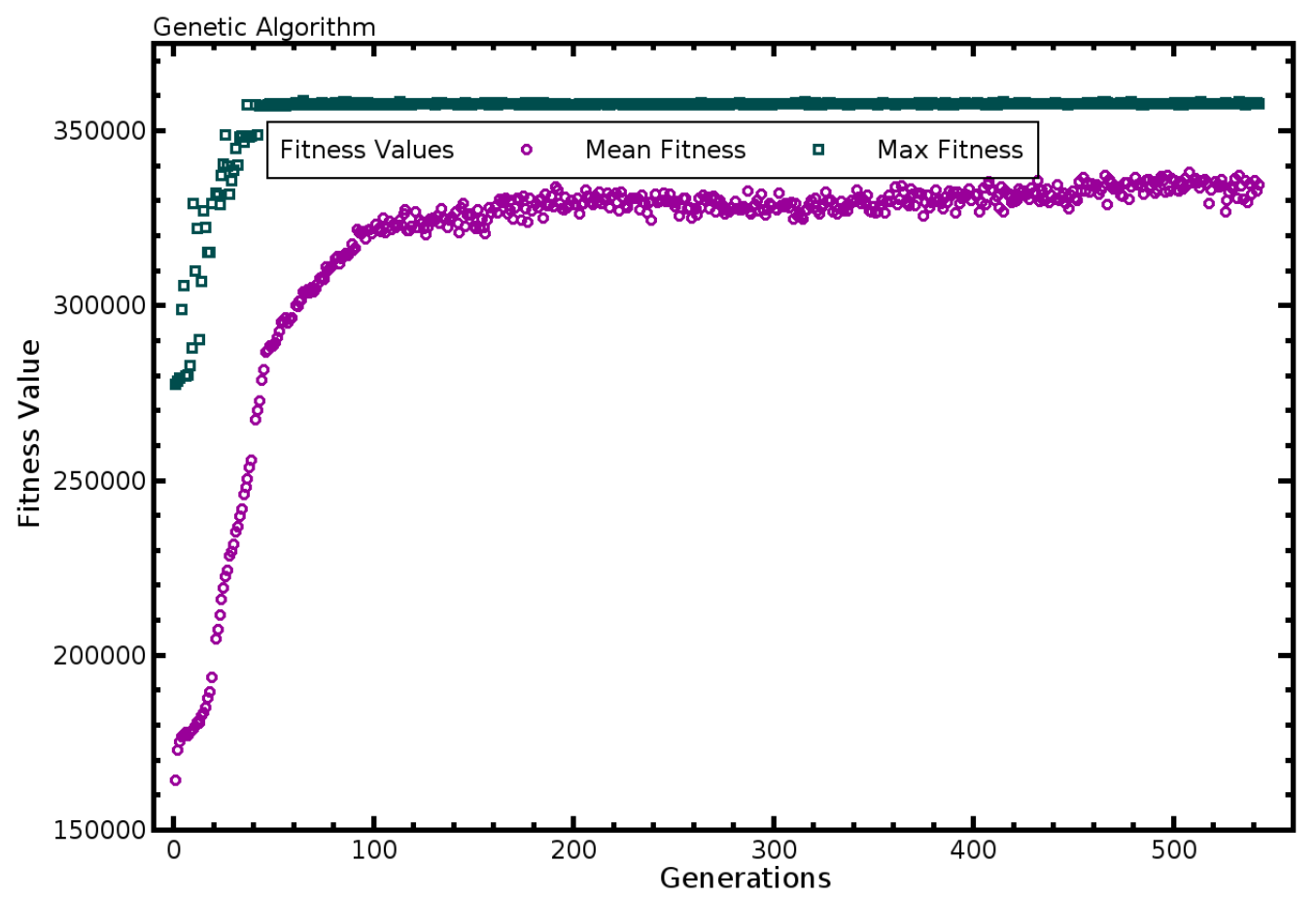

Fig. 2. Maximum and mean values of the fitness values ( $\mathrm{Y}$ axis) obtained by the individuals in each generation ( $\mathrm{X}$ axis) during the run of the genetic algorithm

to facilitate the structure of a Braitenberg vehicle (see Section 2.1). The robot has two ears and two motors. The difference of velocity produces rotation, the same mechanism used in two-wheel robots and emulators. Motor neurons feed motors in a integration fashion, calculating the mean of time passed since motor neurons sent spike to motors. The lower the mean, the faster the wheel.

\subsection{The auditory system of TOPOS}

Mammals process sound signals by means of the auditory system in conjunction with the nervous system $[24,25]$. The mammalian auditory system has three parts that transform sound waves into input signals for the nervous system. The external ear modifies the signal that comes from the sound sources depending on the hearing angle and funnels waves into the auditory channel causing the vibration of the tympanic membrane. The middle ear transfers that movement of the membrane through a chain of bones (malleus, incus, and stapes) to the oval window of cochlea determining the dynamic range of the sound. Finally, the oval window causes a movement of fluid in cochlea, ultimately resulting in stimulation of cochlear hair cells which excite neurons of spiral ganglion that send spike coded auditory signals to the brain through the cochlear nerve.

The symmetric structures of the human brain that estimate the azimuth of 


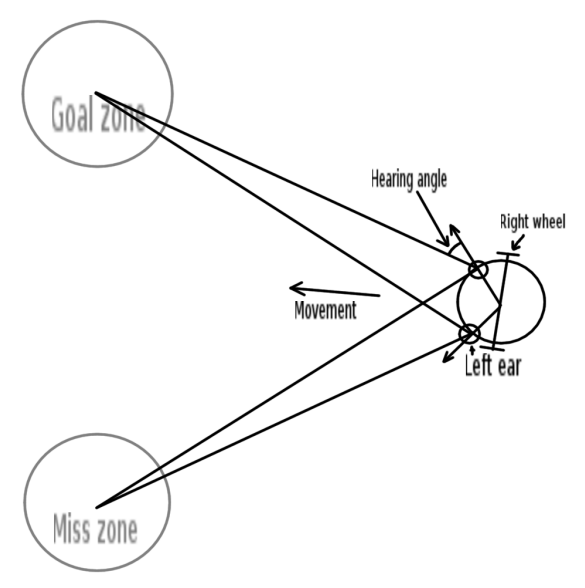

Fig. 3. Process of hearing.

the incoming sound are still under study. It is known that the superior olivary nucleus of brain performs this task. The medial superior olive measures the time differences (important when perceiving low frequencies) and the lateral superior olive measures the level differences for high frequency sounds $[27,28]$. Some results of the work presented in this paper could be compared to these structures.

The acquisition of sound is simulated through the modelling of a cardioid microphone. Thus, the physics of a real microphone is included in the model applying a cardioid-shape function to the received signal as a way to represent the pinna (external ear). The sound is dampened multiplying the amplitude of every frequency (in the simplest scheme) proportionally to the hearing angle (multiplied by 0.0 if sound comes from the back, and by 1.0 if from the front). The emulation of a microphone will allow for an easy embodiment in a real robot. These two cardioid microphones that act like external ears have an angle (one to the right and one to the left) from the front-rear axis of the robot, as can be seen in the figure 3 . This angle is genetically determined as a part of the structure of sensors.

The simulation could add more details, e.g. the shape and materials of the robots that can modify the received sound with delays and filters, but these were discarded to decrease the complexity and the runtime of the simulations.

If we simplify non-linear effects, we can say that the cochlea performs a Fourier Transform of the sound waves, and this is basically the input received by the nervous system [24]. The Fourier Transform takes a wave over time (i.e. a sound sample) as input and produces the vector of complex numbers representing the amplitude and phase of each interval of frequency (the spectrum). In sound-processing computer programs we can see sound in this type of representation (figure 4). Topos uses a proportional scale, but a logarithmic one could be used (musical notes) by simply converting the samples to this scale. 


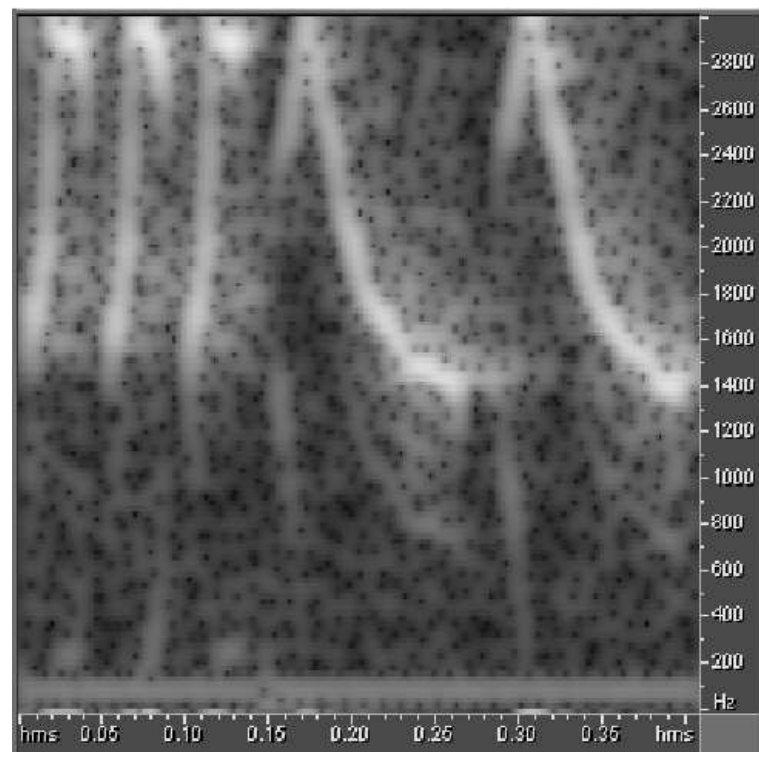

Fig. 4. The frequency spectrum of a bird chirp. Time in seconds (horiz), frequency in $\mathrm{Hz}$ (proportional scale, vert), and highest amplitudes in yellow.

The sound sensors select values from the Fourier Transforms to feed the neurons with their genetically defined activation weight. The sensors are an abstraction of the ones described in [29] (see the figure 5). The Fast Fourier Transform Algorithm divides the frequency spectrum $(3 \mathrm{kHz})$ in 64 proportional strips, because this algorithm needs a power of two.

Each sensor reads information from a strip of the range of frequencies (its characteristic frequency) and takes the values from adjacent strips with less weight. Each strip has a range of $47 \mathrm{~Hz}$, so a deep bass sound is represented in the first strip. This sound will excite a sensor whose central frequency is the second strip, but with less intensity. The value of $3 \mathrm{kHz}$ as the width of the frequency spectrum is the Nyquist frequency, half the minimum $(6 k \mathrm{~Hz})$ sampling rate allowed by the common sound-processing programs [26]. The reason is simple: the lower the sample rate, the lower the computational cost because of the runtime of simulations.

The sensors can react weakly or strongly to close frequencies to the central frequency, depending on the genetic design. There are "wide" and "narrow" sensors, depending on wether they have a high value in this parameter. The sensors also have a threshold and saturation level. These parameters define a $V$-shape line similar to that in figure 5 where real data can be seen. This figure is a biological inspiration to design the sound sensors used in Topos. 


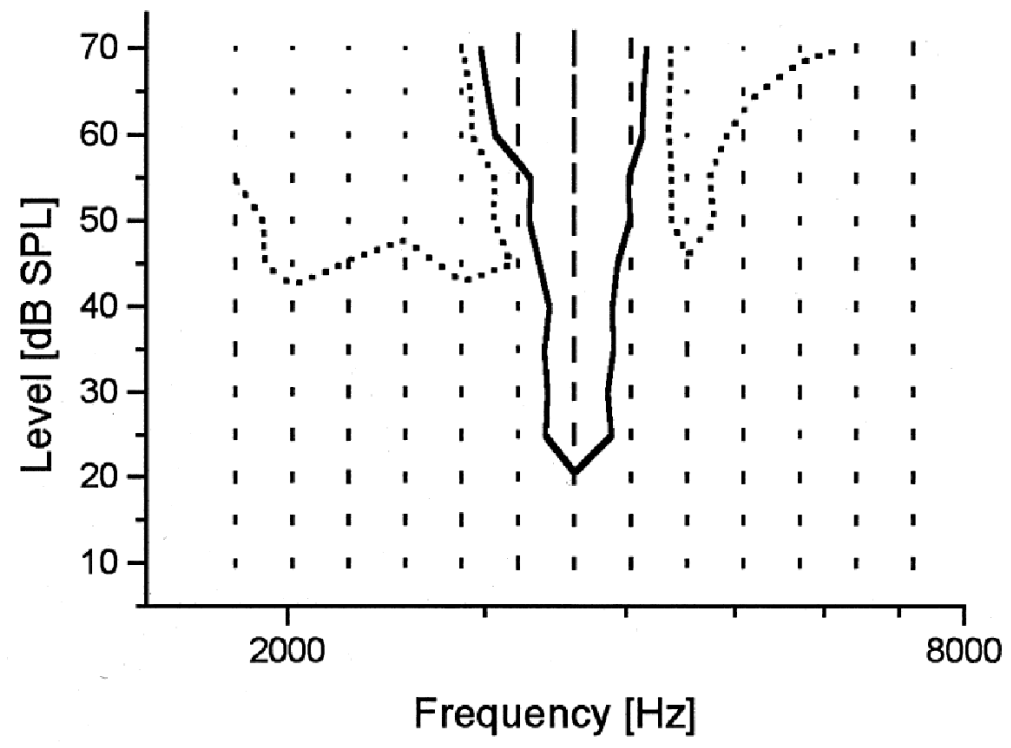

Fig. 5. Experimental data measured with electrodes implanted in starlings. The solid line shows the activation threshold for each frequency. The neuron reacts higher when frequency and amplitude draw a point in the area of the solid line. Figure and caption taken from [29]. Original caption: Example of a response matrix defining the tuning characteristics. The height of the bars indicates the number of impulses per frequency-level combination. An FTC (solid line) and inhibitory side-bands (dotted lines) are added according to the threshold criteria described in the text.

\subsection{Spiking Neural Networks for temporal pattern recognition in complex sounds}

With the sensors described earlier, the robot has to cope with a large amount of dynamical information. For this task and in order to resemble the structure and behaviour of real neurons, we have chosen to develop a type of spiking neurons.

Beer's and others' work in evolutionary robotics use Continuous Time Recurrent Neural Networks (CTRNN) [30,19]. As can be seen in formula 1, each neuron $i$ has a value $\tau_{i}$ to control its time rate of variation. The function $\sigma$ quantifies the firing rate of the neuron.

$$
\dot{y}_{i}=\frac{1}{\tau_{i}} \cdot\left(-y_{i}+\sum_{j=1}^{N} w_{j i} \cdot \sigma\left(y_{j}+\theta_{j}\right)+I_{i}\right) \quad \sigma(x)=\frac{1}{1+e^{-x}}
$$

We also use a recurrent model but with a spiking-type of neurons that are able to code the information in pulse trains and time delays and has a fixed strength spike when the potential of the neuron overflows the threshold.

Spikes travel through axons with a speed and time delay until they reach the 


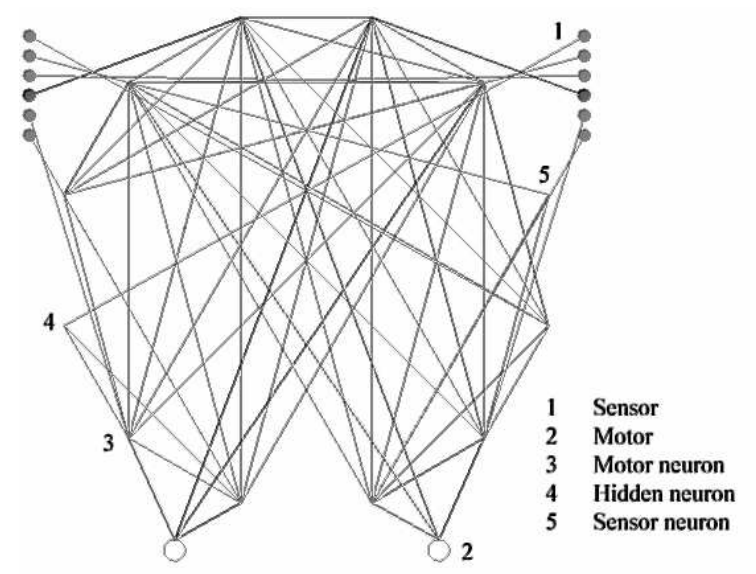

Fig. 6. The internal structure of an individual is shown.

weighted synapses. Inhibitory synapses can block the spike in the postsynaptic neuron. The synapses do not retain the value so the only mechanisms to delay the answer to the stimuli are axon delays and cycles of the network.

The dynamics of the neuron activation can be expressed as a function of time that starts when the sum of weighted synapses overflows the threshold. It has (as the formula 2 shows, being $\boldsymbol{a}_{\boldsymbol{t}}$ the description of a spike and $\boldsymbol{t}$ the time, and $\boldsymbol{\alpha}$ and $\boldsymbol{\beta}$ positive coeficients) a part of exponential attenuation and a part of oscillation that allows for a refractory period.

$$
a_{t}=e^{-\alpha t} \cdot \cos (\beta t)
$$

The spiking neural network implemented in Topos has only recently been used in evolutionary robotics [31-33]. However it has been used in other works under the name of Pulsed Neural Networks [34].

The topology of the artificial neural network is shown in figure 6. Two fullyrecurrent subnets are symmetric and interconnected.

These artificial neural networks show a rich variety of behaviour (including chaotic dynamics) [30]. The most important characteristics of these networks are the following: they can integrate perceptions over time before actions [35]; they are biologically plausible [36]; they can process time information (delay among stimuli); and they are mathematically equivalent to sigmoid networks, more robust to noise, and sometimes requiring significantly fewer neurons [32]. 


\section{Experiments}

An experiment is a run of the genetic algorithm given two sounds and a initial population of randomly-generated robots. For a generation, each robot is evaluated in a number of trials and gets a fitness value (see section 3 ). At the end of the run of each experiment, an elite formed by $25 \%$ of individuals with better fitness is selected and their efficiency in solving the problem is measured and represented in a graphic that shows the ability of the best robots of the population to identify a given sound, navigating towards it. Two histograms describe each experiment, one for EFA and one for EFR. These two variables measure the ability of each robot to distinguish the two sources, instead of the fitness used for evolution:

- absolute effectiveness $(E F A)=$ hits/trials

- relative effectiveness $(E F R)=$ hits $/$ (hits + misses $)$

A hit occurs when a robot reaches a circle around the correct source of a given radius (the distance between sources divided by 4 ), and a miss when the individual crosses the frontier of the incorrect source.

\subsection{Some experiments that prove the correctness of the system}

To prove that the Topos system works correctly and to set some basic results as a test collection, we prepared some experiments (from left to right and from up to bottom in figure 7):

Dependency of the stimuli (Experiment SS): the absolute and relative efficiency are 0 for all the individuals if both sources are Silent. The conclusion is that the robots are able to navigate only if they have a sound stimulus.

Positive phonotaxis (Experiment MS): the population evolves to identify a sample of Music in one source, the second being Silent. The source they have to reach is the Music (the first letter in the two-letter code). We obtained the typical behaviour of a type of Braitenberg vehicles [4] (positive phonotaxis) with absolute and relative efficiency near $100 \%$.

Incapability to distinguish identical sounds (Exps. MM and WW): in this case the same sample of Music is used for the two sources, and the starting point of the sample is randomly set at each source. The robots have to get closer to the correct source, but since both sources emit the same sound there is no clue to navigate. The consequence is a low score of hits and as many hits as fails. Most individuals (30\%) in the elite obtain $0 \%$ of efficiency. The WW experiment is basically the same as the MM experiment but the two sources emit White noise. The relative efficiency follows a 

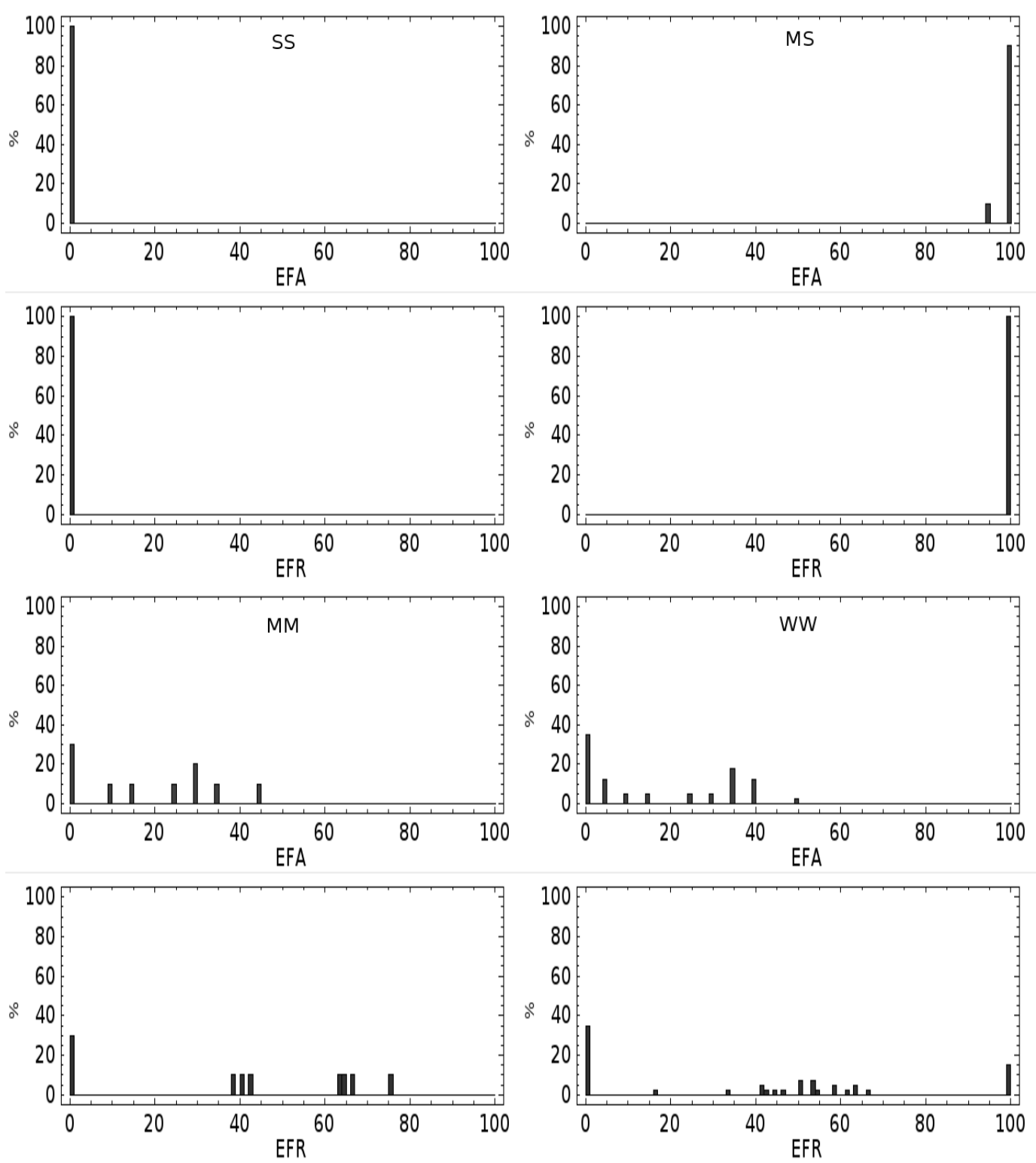

Fig. 7. Four basic experiments to test the correctness of the system. Two histograms for each experiment, the upper representing the EFA and the lower showing the EFR. The histograms represent the amount of individuals in the last elite that get that percentage of efficiency. Analysis in the text.

normal distribution with mean in $50 \%$ of hits and misses, plus some robots with EFA and EFR equal to zero. As in the previous experiment, there is a number of robots that do the task sometimes and get high scores in EFR but low in absolute effectiveness (up to $35 \%$ gives no correct answer or simply no answer, that is, EFA is zero).

These experiments show that the robots need the ability to differentiate the sounds to perform the task of navigation towards the correct source. 

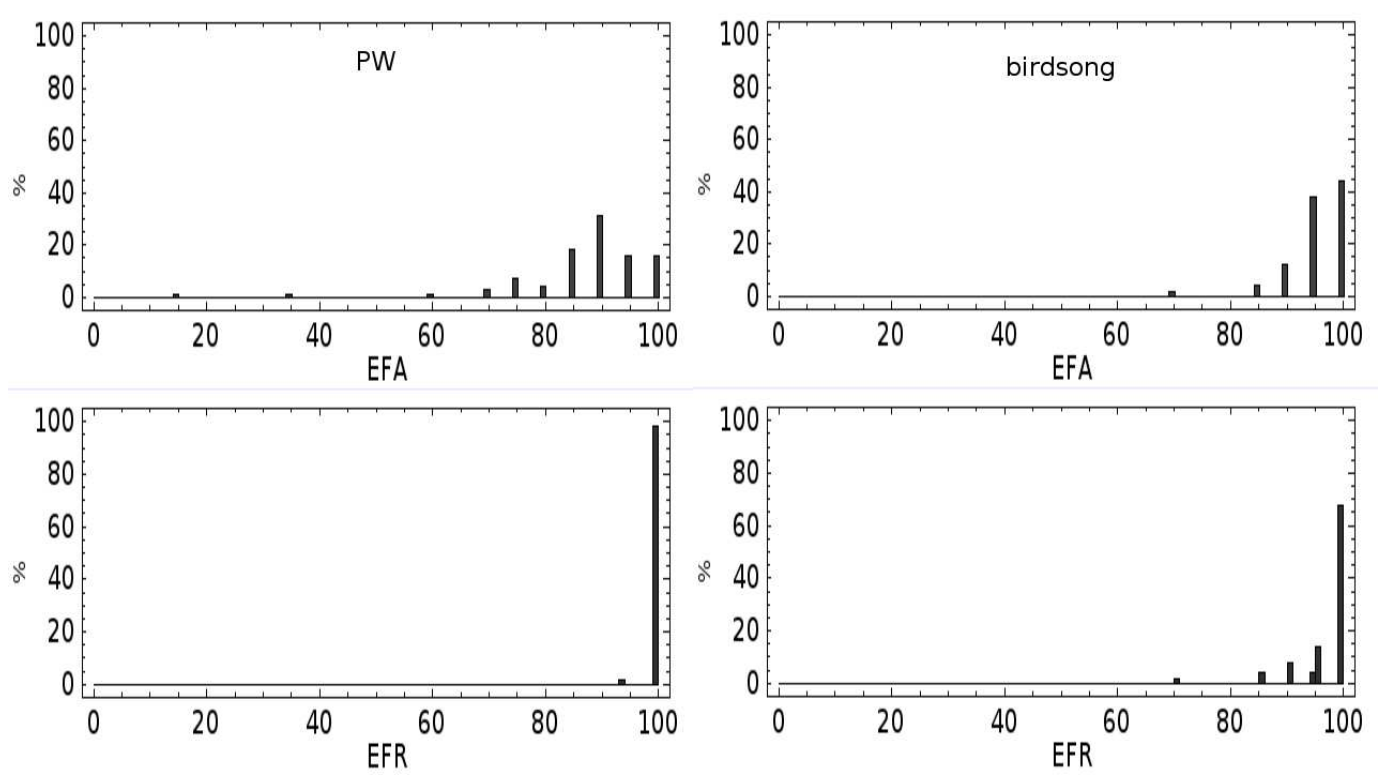

Fig. 8. Histograms for the experiment that uses pink noise and white noise, on the left; histograms of the experiments with two birdsongs from the figure 9 , on the right.

\subsection{The efficiency of Topos to recognise complex signals}

The experiments described in Section 4.1 prove the correctness of the Topos system. Then, the ability of the individuals to recognise complex signals (i.e. the sounds found in nature) is analysed.

Pink noise is a common natural sound. In figure 8 the results of the PW experiment are presented. The experiment evolves a population to navigate towards a source of pink noise and avoiding a source of white noise. As can be seen, adapted individuals (result of the evolution) are able to correctly select the pink source with an efficiency score close to $100 \%$. Relative effectiveness of $100 \%$ means the absence of errors. An experiment in which the robots have to avoid the pink noise and reach the white noise produces the same results. Other experiments with two slightly different parts of a rock song also produce good results.

The PW experiment also shows that the robots can choose the pink noise, a sound with less amplitude for most of the frecuencies, and avoid the louder white noise.

In a second experiment, the sounds are real canary-bird chirps. We have used parts of a birdsong as another usual sound in nature, but any type of sound could be chosen. The two samples sound simultaneously but the starting point is selected at random. The robots could use the "trick" of discrimination by filtering frequencies with the sensors, as some animals do (and probably used 


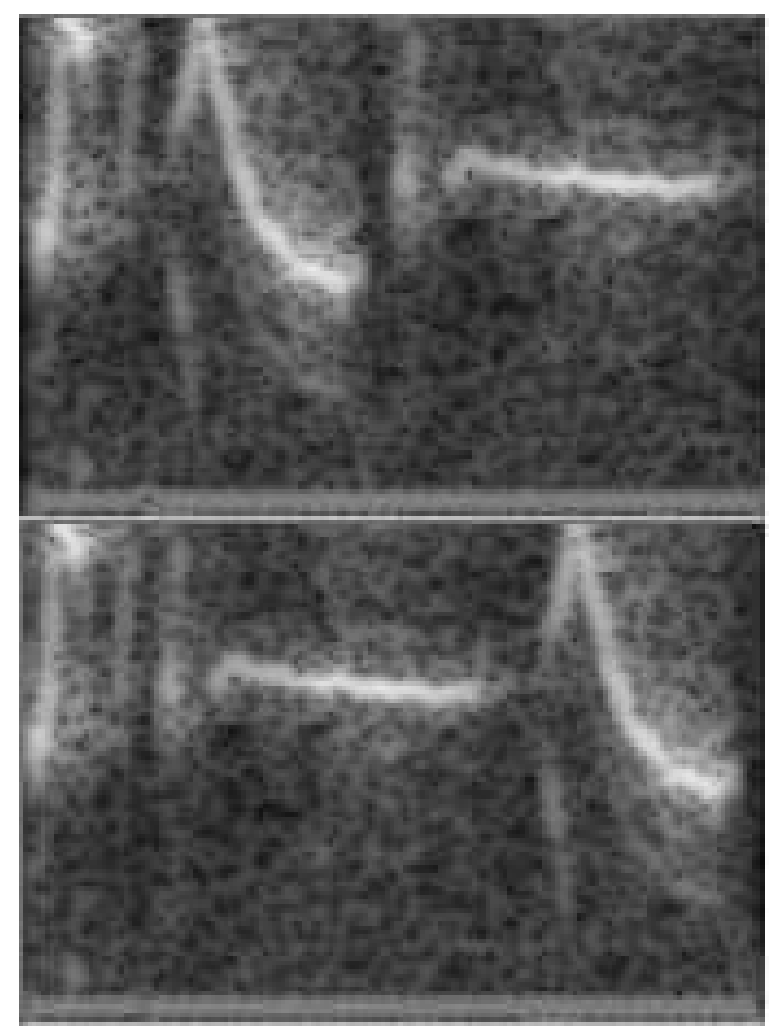

Fig. 9. The frequency spectrum of the sounds prepared from a bird chirp. The experiment prepared to test the Topos system uses the upper sequence in a sound source and the lower in the other source. Note that the only difference is the order in its parts, permuted like ABC and ACB, or in words "per" and "pre". Time in seconds (horiz), frequency in $\mathrm{Hz}$ (vert), and highest amplitudes in yellow.

in the PW experiment). An example is that crickets only react to chirps from their own species [37]. In order to avoid this trick, we use the same sound but with a different order in its parts, like $\mathrm{ABC}$ and $\mathrm{ACB}$ (see figure 9). Figure 8 shows the high scores of absolute and relative efficiency of the system in recognising such a complex signal. Almost a half of the elite performs perfectly (EFA is 100\%) and nearly all of them (more than $80 \%$ ) are able to recognise the correct sound. The behaviour of the robots when performing the navigation task can be seen in figure 1, and the fitness values in the genetic algorithm in figure 2 .

\subsection{Robustness to background noises}

Background noises interfere with the intelligibility and ease of perceiving sounds. These experiments show the robustness to sounds that perturb the recognition of the birdsongs used in previous experiment.

In the first of these experiments there are two additional sounds emitted from 

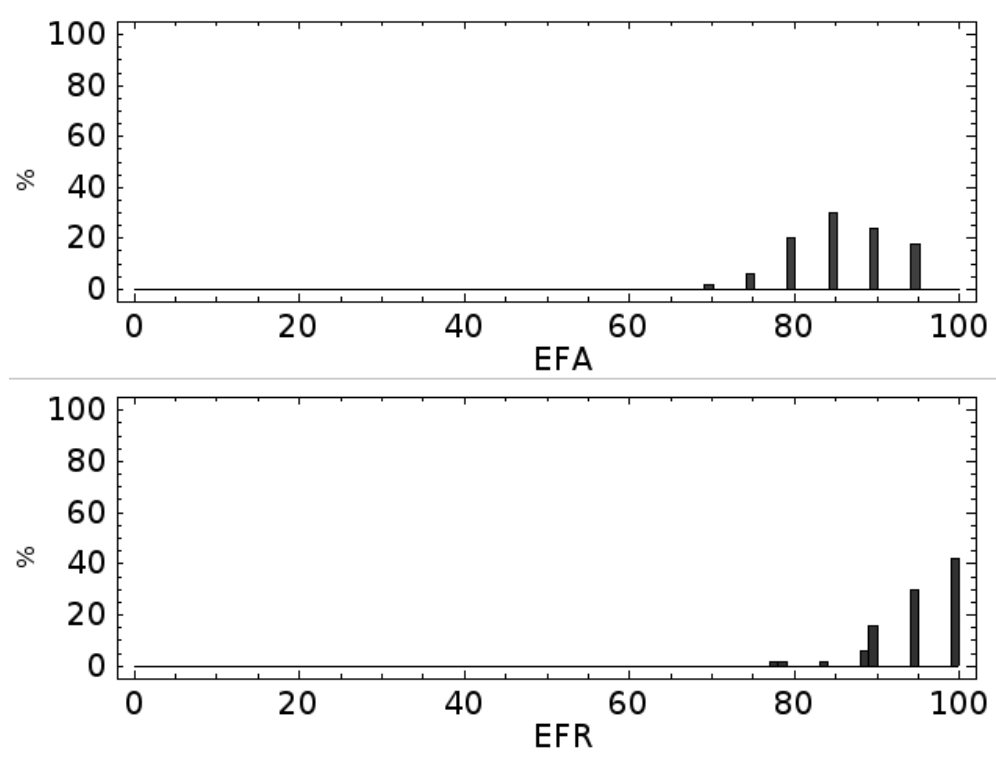

Fig. 10. Histograms for the experiment that has additional background sounds composed of drums and rock music.

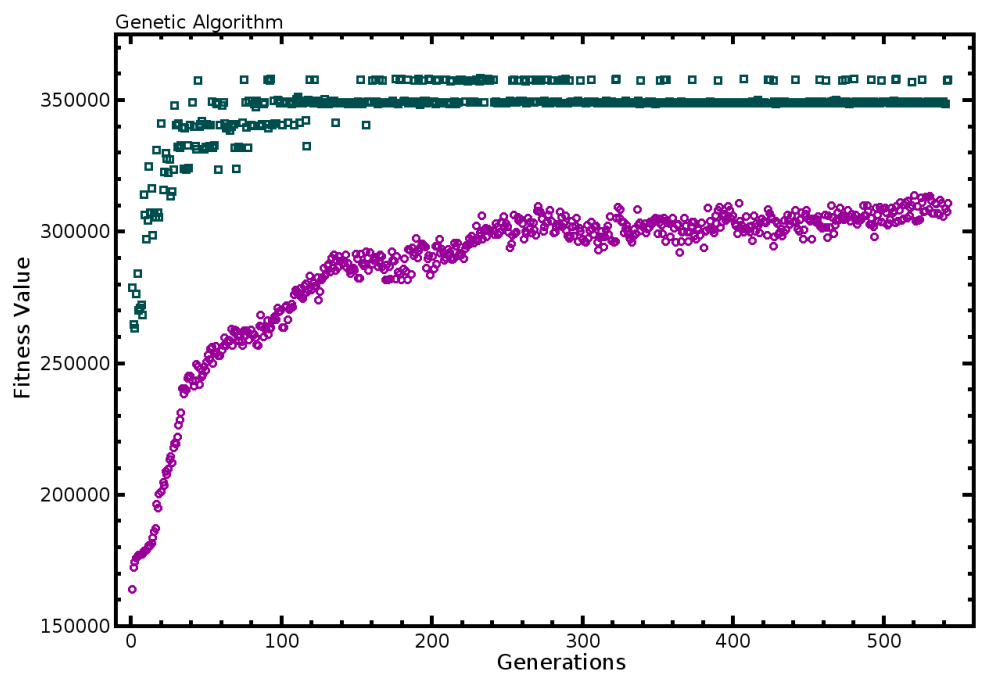

Fig. 11. Maximum and mean values of the fitness values obtained by the individuals in each generation during the run of the genetic algorithm for the experiment that has background noises. Compare to figure 2 .

random places (near to the main sources) during each trial. These sounds are composed of a number of different drums and rock music.

In figures 10 and 11 the data reveals that it is more difficult for the robots to distinguish the two birdsongs, comparing to the histograms on the right in figure 8 and 2. They are able to achieve it most of the times but not always due to the perturbation that prevents the hearing. A $85 \%$ of the trials they manage a hit (EFA), and the proportion between hits and misses (EFR) is not as good as in the second experiment (section 4.2). 

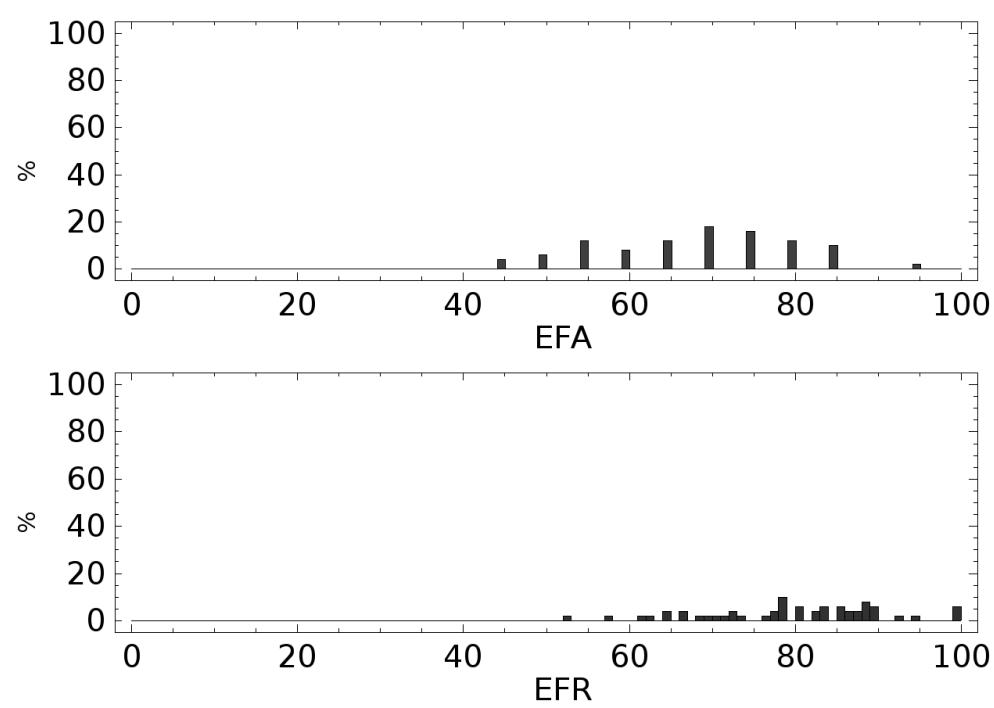

Fig. 12. Histograms for the experiment that has two additional sources of white noise.
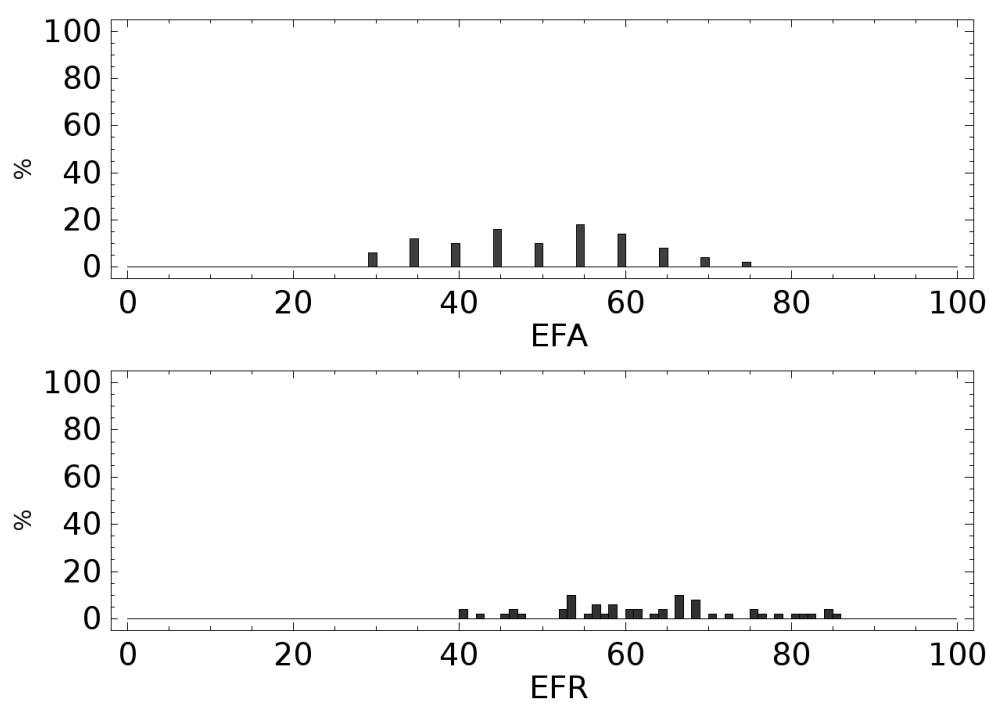

Fig. 13. Histograms for the experiment that has four additional sources of white noise.

The figures 12 and 13 show that the ability to perform the task decreases as the number of additional sources of white noise increases. It is more difficult, but not impossible, to distinguish between the birdsongs when the background noises are white noise, compared to the ones composed of music and drums (see figure 10), but the robots get quite good scores and try to perform the task (compare to figure 7). 


\section{Conclusions}

This paper describes the Topos application and the considerations that have been made to build this simulation of two-wheel robots. Evolutionary Robotics techniques allow to obtain the embodied control system of the robot by evolution. The control system is composed of biologically inspired artificial cochleas and spiking neural networks. The embodied system has physical grounded symbols because the simulation is based on the nouvelle artificial intelligence. Some experiments confirm the validity of the approach through the study of the ability of the robots to distinguish between real canary songs. The system could be applied to any type and combination of dynamical signals, since they can distinguish signals using frequencies and time characteristics.

Due to the problem of overconvergence in genetic algorithms, the robots learn too much of what to do, that their neural networks lose the capacity of generalising and recognising other sounds than learnt ones. A larger set of training couples of sounds could solve this problem but requires a much longer runtime. Also, a set of different test sounds with a working robot (that can distinguish between the training sounds) would allow to detect the characteristics used to perform the recognition.

This system broadens the perspective both from the technical and epistemic points of view, since it is possible to develop such a robot and we have gained further information to study cognition. The functioning of the neural network is an interesting and difficult area. The work in progress shows that the delays and weights in axons and the position and shape of the sensors can be what the robots need in order to discriminate the sounds.

In real world robot applications, motor and other background noises have a severe impact on auditory recognition. This work has discovered that adding background noises has little impact on robot navigation and the fitness of its population, and that noise has hardly any impact at all.

Further work is the research of the addition to the model of noises from the motors of the robot, since it is a way to implement the propioception. This interesting topic deserves time and careful research because the hypothesis that has to be rigorously proven, is that propioception improves navigation.

Two basic points to deal with in a further development of the system are, on the one hand, the computational costs of the trials (due to the complexity of the simulation) through the run of the genetic algorithm, with some trials per individual (from ten to twenty trials) and thousands of complex robots each generation (up to ten minutes per generation, it takes days of a powerful computer); and on the other hand, the morphogenesis or the difficulty of evolving such a complex structure more than the variation in parameters (e.g. 
the shape of the robot), and maybe the need of a non-linear expression of the information coded in the genome. This is a common and generic problem of scalability in evolutionary robotics.

\section{References}

[1] J. Holland, Adaptation in Natural and Artificial Systems, U. Michigan Press, 1975 .

[2] D. E. Goldberg, Genetic Algorithms in Search, Optimization and Machine Learning, Addison-Weley, Reading, 1989.

[3] S. J. Russell, P. Norvig, Artificial Intelligence: A Modern Approach, Prentice Hall, 1994.

[4] V. Braitenberg, Vehicles. Experiments in Synthetic Psychology, MIT Press, MA, 1984 .

[5] C. G. Langton (Ed.), Artificial Life: the proceedings of an interdisciplinary workshop on the synthesis and simulation of living systems, Addison-Wesley, Redwood City, CA, 1989, workshop held September, 1987 in Los Alamos, New Mexico.

[6] C. Langton, C. Taylor, J. D. Farmer, S. Rasmussen, Artificial Life II, Redwood City, CA: Addison Wesley, 1991.

[7] J.-A. Meyer, S. W. Wilson (Eds.), From Animals to Animats: Proceedings of the 1st International Conference on Simulation of Adaptive Behavior, MIT Press, Cambridge, MA, 1991, Paris.

[8] R. Brooks, Elephants don't play chess, Robotics and Autonomous Systems 6 (1990) 3-15.

[9] R. Brooks, Intelligence without representation, Artificial Intelligence 47 (1991) 139-159.

[10] R. Brooks, Intelligence without reason, in: J. Myopoulos, e. Ray Reiter (Eds.), Proc. 12th IJCAI, Morgan Kaufmann publishers Inc.: San Mateo, CA, 1991, pp. 569-595.

[11] R. Beer, The dynamics of active categorical perception in an evolved model agent, Adaptive Behavior 11(4) (2003) 209-243.

[12] I. Harvey, P. Husbands, D. Cliff, Issues in evolutionary robotics, in: R. H. Meyer, J.-A., S. Wilson (Eds.), 2nd Intl. Conf. on Simulation of Adaptive Behavior, SAB2, MIT Press/Bradford Book, 1992, pp. 364-373.

[13] D. Cliff, I. Harvey, P. Husbands, Explorations in evolutionary robotics, Adaptive Behavior 2(1) (1993) 71-108. 
[14] I. Harvey, The artificial evolution of adaptive behaviour. Ph.D. thesis, University of Sussex. (1995).

[15] J. R. Koza, Genetic programming: on the programming of computers by means of natural selection, MIT Press, Cambridge, MA, 1992.

[16] R. Beer, J. C. Gallagher, Evolving dynamical neural networks for adaptive behavior, Adaptive Behavior 1(1) (1992) 91-122.

[17] D. Floreano, F. Mondada, Automatic creation of an autonomous agent: Genetic evolution of a neural-network driven robot, in: D. Cliff, P. Husbands, J. Meyer, S. Wilson (Eds.), From Animals to Animats III: SAB'94, MIT Press-Bradford Books, Cambridge, MA, 1994.

[18] I. Harvey, P. Husbands, D. Cliff, Seeing the light: Artificial evolution, real vision, in: P. H. David Cliff, J.-A. Meyer (Eds.), From Animals to Animats 3. Proceedings of the Third International Conference on Simulation of Adaptive Behavior, MIT Press, Cambridge, MA, 1994, pp. 392-402.

[19] I. Harvey, E. Di Paolo, R. Wood, M. Quinn, E. A. Tuci, Evolutionary robotics: A new scientific tool for studying cognition, Artificial Life 11(1-2) (2005) 79-98.

[20] P. González-Nalda, B. Cases, Topos: generalized Braitenberg vehicles that recognize complex real sounds as landmarks, in: L. Rocha, L. Yaeger, M. Bedau, D. Floreano, R. Goldstone, A. Vespignani (Eds.), Alife X: 10th International Conference on the Simulation and Synthesis of Living Systems, Cambridge, MIT Press, 2006, pp. 213-219.

[21] P. González-Nalda, B. Cases, Topos: reconocimiento de patrones temporales en sonidos reales con redes neuronales de pulsos, in: A. Fernández-Caballero, M. Gracia Manzano, E. Alonso, S. Miguel Tomé (Eds.), Una perspectiva de la Inteligencia Artificial en su 50 aniversario, Universidad de Castilla-La Mancha, 2006, pp. 243-248.

[22] B. F. Skinner, The behavior of organisms: An experimental analysis, New York: Appleton-Century, 1938.

[23] P. Husbands, I. Harvey, D. Cliff, G. Miller, The use of genetic algorithms for the development of sensorimotor control systems, in: P. Gaussier, J.-D. Nicoud (Eds.), Proceedings of the From Perception to Action Conference (PerAc'94), IEEE Computer Society Press, 1994, pp. 110-121.

[24] S. Handel, Listening: An Introduction to the Perception of Auditory Events, The MIT Press, Cambridge, MA, 1989.

[25] B. C. J. Moore, An Introduction to the Psychology of Hearing, 4th Ed. Academic Press, London, 1997.

[26] H. Nyquist, Certain Topics in Telegraph Transmission Theory, A.I.E.E. Trans. 47 (1928) 617.

[27] E. R. Kandel, J. Schwartz, T. M. Jessell, Principles of Neural Science, Mc Graw Hill, 2000. 
[28] H. Gray, Anatomy of the Human Body (Gray's Anatomy), Philadelphia: Lea \& Febiger, 1918.

[29] A. Nieder, G. Klump, Adjustable frequency selectivity of auditory forebrain neurons recorded in a freely moving songbird via radiotelemetry, Hearing Research 127 (1999) 41-54.

[30] R. Beer, On the dynamics of small continuous-time recurrent neural networks, Adaptive Behavior 3(4) (1995) 469-509.

[31] E. A. Di Paolo, Evolving spike-timing dependent plasticity for single-trial learning in robots, Philosophical Transactions of the Royal Society A. 361 (2003) 2299-2319.

[32] W. Maass, Networks of spiking neurons: the third generation of neural network models, Neural Networks 10 (1997) 1659-1671.

[33] D. Floreano, C. Mattiussi, Evolution of spiking neural controllers for autonomous vision-based robots, in: T. Gomi (Ed.), Evolutionary Robotics IV, Berlin, Springer-Verlag, 2001, pp. 3-10.

[34] W. Maass, C. M. Bishop (eds.), Pulsed Neural Networks, MIT Press, 1999.

[35] B. Yamauchi, R. Beer, Integrating reactive, sequential, and learning behavior using dynamical neural networks, in: D. Cliff, P. Husbands, J. Meyer, S. Wilson (Eds.), From Animals to Animats III: International Conference on Simulation of Adaptive Behaviour SAB'94, MIT Press-Bradford Books, Cambridge, MA, 1994, pp. 382-391.

[36] D. Floreano, Y. Epars, J. Zufferey, C. Mattiussi, Evolution of Spiking Neural Circuits in Autonomous Mobile Robots, International Journal of Intelligent Systems 21.

[37] H. H. Lund, B. Webb, J. Hallam, A robot attracted to the cricket species gryllus bimaculatus, in: P. Husbands, I. Harvey (Eds.), IV European Conference on Artificial Life ECAL97, MIT Press/Bradford Books, MA, 1997, pp. 246-255. 